\title{
Clinical audit to improve patient care
}

\author{
Ping Yein Lee \\ Deputy Chief Editor \\ Lee PY. Clinical audit to improve patient care. Malays Fam Physician. 2021;16(2);1. https://doi.org/10.51866/ed0002
}

In this issue of MFP, two of the original articles are related to clinical audit. ${ }^{1-2}$ This highlights the importance of clinical audit in improving the quality of patient care. ${ }^{3}$ Clinical audit is a process that measures the quality of care provided in a healthcare setting against relevant standards. Subsequently, this involves the implementation of interventions to improve the quality of care. ${ }^{3}$ An effective clinical audit process involves a cycle of activities that comprises the following steps: ${ }^{3-4}$

1) Selecting standards - This involves either setting a custom standard or adopting existing standards or guidelines.

2) Performing the audit - This involves analysing the data from previous records or performing ongoing monitoring to identify gaps in meeting the standards.

3) Identifying the factors contributing to gaps in meeting the standards - This involves identifying the barriers and facilitators to improving care.

4) Implementing the intervention - This involves setting priorities and taking actions to improve what can be done in the setting.

5) Re-auditing - This involves checking whether the quality of care has been improved.

6) Repeating the audit cycle - If there is no improvement, it is important to find other solutions and repeat the audit cycle. If there is an improvement, repeating the audit cycle will identify solutions for another set of problems. Alternatively, measurements can be made against a different set of standards for further improvement.

For the article on clinical audit of the management of smear-positive adult pulmonary tuberculosis by Cumarasamy et al. ${ }^{1}$, the study reported up to the findings of the gaps in meeting the standard of care in terms of the guidelines and recommendations on factors for improving care. However, article by Low et $\mathrm{al}^{2}{ }^{2}$ on the effectiveness of simple interventions to remind eye doctors to educate glaucoma patients, had manage to report a full audit cycle and showed improvements in patient care. To achieve the ultimate aim of improving the quality of care in clinical practice, the clinical audit process should follow a full audit cycle. Additionally, the sustainability of improvement or change from the audit process is also essential. This should include plans to: 1) monitor and evaluate the change as well as 2) maintain and reinforce the change. ${ }^{3}$

\section{References}

1. Cumarasamy R, Azmi FS, Salamon N. Clinical audit of management of smear positive adult pulmonary tuberculosis in PR1 tuberculosis clinic, KK Bandar Miri. Malays Fam Physician. 2021;16(2);50-57. Available from: https://doi. org/10.51866/oa1215.
2. Low ASL, Singh S, Ang EL, et al. Effectiveness of simple interventions to remind eye doctors to educate glaucoma patients about the dangers of driving. Malays Fam Physician. 2021;16(2);45-49. Available from: https://doi. org/10.51866/oa1147.
3. National Institute for Clinical Excellence. Principles for Best Practice in Clinical Audit [Internet]. Oxon, UK: Radcliffe Medical Press; 2002 [cited 2021 May 17]. Available from: http://www.nice.org.uk/media/796/23/Best PracticeClinicalAudit.pdf.

4. Yorston D, Wormald R. Clinical auditing to improve patient outcomes. Community Eye Health. 2010;23(74):48-9. 